\title{
The Classification of the Applicable Machine Learning Methods in Robot Manipulators
}

\author{
Hadi Hormozi, Elham Hormozi, and Hamed Rahimi Nohooji
}

\begin{abstract}
Supervised machine learning is the search for algorithms that reason from externally supplied instances to produce general hypotheses, which then make predictions about future instances. In other words, the goal of supervised learning is to build a concise model of the distribution of class labels in terms of predictor features. The resulting classifier is then used to assign class labels to the testing instances where the values of the predictor features are known, but the value of the class label is unknown. This paper describes various supervised machine learning classification techniques used in robotic manipulators. Of course, a single article cannot be a complete review of all supervised machine learning classification algorithms (also known induction classification algorithms), yet we hope that the references cited will cover the major theoretical issues, guiding the researcher in interesting research directions and suggesting possible bias combinations that have yet to be explored.
\end{abstract}

Index Terms-Machine learning, adaptive control, repetitive control, robot manipulators.

\section{INTRODUCTION}

Machine learning, a branch of artificial intelligence, is a scientific discipline that is concerned with the design and development of algorithms that allow computers to evolve behaviors based on empirical data [1]. A major focus of machine learning research is to automatically learn to recognize complex patterns and make intelligent decisions based on data; the difficulty lies in the fact that the set of all possible behaviors given all possible inputs is too large to be covered by the set of observed examples (training data). Hence the learner must generalize from the given examples, so as to be able to produce a useful output in new cases. Machine learning, like all subjects in artificial intelligence, require cross-disciplinary proficiency in several areas, such as probability theory, statistics, pattern recognition, cognitive science, data mining, adaptive control, computational neuroscience and theoretical computer science [2]. In this paper we are focused on learning algorithms for robot manipulators.

\section{Machine LeARning AlgorithmS}

Machine learning algorithms are organized into a taxonomy based on the desired outcome of the algorithm.

Supervised learning generates a function that maps inputs to desired outputs. For example, in a classification problem,

Manuscript received May 30, 2012.

The authors are with the Department of Computer Science, Islamic Azad University, Buinzahra branch, Buinzahra, Iran(e-mail: hadyhormozy@yahoo.com). the learner approximates a function mapping a vector into classes by looking at input-output examples of the function for robot manipulators [1].

Unsupervised learning models a set of inputs, like clustering [1].

Semi-supervised learning combines both labeled and unlabeled examples to generate an appropriate function or classifier in manipulators [3].

Reinforcement learning learns how to act given an observation of the world. Every action has some impact in the environment, and the environment provides feedback in the form of rewards that guides the learning algorithm [1] and [3].

Transduction tries to predict new outputs based on training inputs, training outputs, and test inputs [3].

Learning to learn learns its own inductive bias based on previous experience for robots [1].

Supervised algorithms are more basic than other classifications in manipulating of robots. Because there are several ways in which the standard supervised learning problem can be generalized:

1. Semi-supervised learning: In this setting, the desired output values are provided only for a subset of the training data. The remaining data is unlabeled [4].

2. Active learning: Instead of assuming that all of the training examples are given at the start, active learning algorithms interactively collect new examples, typically by making queries to a human user. Often, the queries are based on unlabeled data, which is a scenario that combines semi-supervised learning with active learning [5].

3. Structured prediction: When the desired output value is a complex object, such as a parse tree or a labeled graph, then standard methods must be extended [6].

4. Learning to rank: When the input is a set of objects and the desired output is a ranking of those objects, then again the standard methods must be extended [7].

\section{TAXONOMY OF SUPERVISED LEARNING ALGORITHMS}

Supervised learning algorithms generally are divided to the following methods:

- Averaged One-Dependence Estimators (AODE)

- Artificial neural network

- Bayesian statistics

- Case-based reasoning

- Decision trees

- Inductive logic programming

- Group method of data handling (GMDH)

- Learning Automata

- Minimum message length (decision trees, decision 
graphs, etc.)

- Lazy learning

- Instance-based learning

- Probably approximately correct learning (PAC)

- Support vector machines

- Information fuzzy networks (IFN)

Averaged One-Dependence Estimators is a probabilistic classification learning technique. It was developed to address the attribute-independence problem of the popular Naive Bayes classifier. It frequently develops substantially more accurate classifiers than naive Bayes at the cost of a modest increase in the amount of computation [2].

An artificial neural network (ANN), usually called neural network $(\mathrm{NN})$, is a mathematical model or computational model that is inspired by the structure and/or functional aspects of biological neural networks [8]. A neural network consists of an interconnected group of artificial neurons, and it processes information using a connectionist approach to computation. In most cases an ANN is an adaptive system that changes its structure based on external or internal information that flows through the network during the learning phase. Modern neural networks are non-linear statistical data modeling tools. They are usually used to model complex relationships between inputs and outputs or to find patterns in data (For example: Back propagation method) [9] and [10].

Bayesian inference is a method of statistical inference in which some kind of evidence or observations are used to calculate the probability that a hypothesis may be true, or else to update its previously-calculated probability. The term "Bayesian" comes from its use of the Bayes' theorem in the calculation process. Bayes' theorem was deduced in several special cases by Thomas Bayes, and then it was extended to the general theorem by other researchers. In practical usage, "Bayesian inference" refers to the use of a prior probability over hypotheses to determine the likelihood of a particular hypothesis given some observed evidence; that is, the likelihood that a particular hypothesis is true given some observed evidence (the so-called posterior probability of the hypothesis) comes from a combination of the inherent likelihood (or prior probability) of the hypothesis and the compatibility of the observed evidence with the hypothesis (or likelihood of the evidence, in a technical sense) [11]. Bayesian inference is opposed to frequentist inference, which makes use only of the likelihood of the evidence (in the technical sense), discounting the prior probability of the hypothesis. Most elementary undergraduate-level statistics courses teach frequentist inference rather than Bayesian inference [12]. Bayesian method has three parts [2] : Naive Bayes classifier [13-15], Bayesian network [16], [17] and Bayesian knowledge base [17], [18].

Case-based reasoning (CBR), broadly construed, is the process of solving new problems based on the solutions of similar past problems. An auto mechanic who fixes an engine by recalling another car that exhibited similar symptoms is using case-based reasoning. A lawyer who advocates a particular outcome in a trial based on legal precedents or a judge who creates case law is using case-based reasoning. So, too, an engineer copying working elements of nature (practicing bio mimicry), is treating nature as a database of solutions to problems. Case-based reasoning is a prominent kind of analogy making. It has been argued that case-based reasoning is not only a powerful method for computer reasoning, but also a pervasive behavior in everyday human problem solving; or, more radically, that all reasoning is based on past cases personally experienced. This view is related to prototype theory, which is most deeply explored in cognitive science [19].

Decision tree learning, used in data mining and machine learning, uses a decision tree as a predictive model which maps observations about an item to conclusions about the item's target value. More descriptive names for such tree models are classification trees or regression trees [20]. In these tree structures, leaves represent classifications and branches represent conjunctions of features that lead to those classifications. In decision analysis, a decision tree can be used to visually and explicitly represent decisions and decision making. In data mining, a decision tree describes data but not decisions; rather the resulting classification tree can be an input for decision making. This page deals with decision trees in data mining [21].

Inductive logic programming (ILP) is a subfield of machine learning which uses logic programming as a uniform representation for examples, background knowledge and hypotheses. Given an encoding of the known background knowledge and a set of examples represented as a logical database of facts, an ILP system will derive a hypothesized logic program which entails all the positive and none of the negative examples [22]. Inductive logic programming is particularly useful in bioinformatics and natural language processing [23]. The term inductive logic programming was first introduced in a paper by Stephen Muggleton [22].

Gaussian process regression (Kriging) is a group of geostatistical techniques to interpolate the value of a random field (e.g., the elevation, z, of the landscape as a function of the geographic location) at an unobserved location from observations of its value at nearby locations [24] and [25]. The theory behind interpolation and extrapolation by kriging was developed by the French mathematician Georges Matheron based on the Master's thesis of Daniel Gerhardus Krige, the pioneering plotter of distance-weighted average gold grades at the Witwatersrand reef complex in South Africa. The English verb is to krige and the most common noun is kriging; both are often pronounced with a hard "g", following the pronunciation of the name "Krige" [24].

Group method of data handling (GMDH) is a family of inductive algorithms for computer-based mathematical modeling of multi-parametric datasets that features fully-automatic structural and parametric optimization of models [26]. GMDH is used in such fields as data mining, knowledge discovery, prediction, complex systems modeling, optimization and pattern recognition. GMDH algorithms are characterized by inductive procedure that performs sorting-out of gradually complicated polynomial models and selecting the best solution by means of the so-called external criterion [27] and [28].

Learning automata a branch of the theory of adaptive control is devoted to learning automata surveyed by Narendra and Thathachar which were originally described explicitly as 
finite state automata. Learning automata select their current action based on past experiences from the environment [29]. A learning automaton is an adaptive decision-making unit situated in a random environment that learns the optimal action through repeated interactions with its environment. The actions are chosen according to a specific probability distribution which is updated based on the environment response the automaton obtains by performing a particular action [30]. With respect to the field of reinforcement learning, learning automata are characterized as policy iterators. In contrast to other reinforcement learners, policy iterators directly manipulate the policy $\pi$. Another example for policy iterators are evolutionary algorithms [31].

Lazy learning: In artificial intelligence, lazy learning is a learning method in which generalization beyond the training data is delayed until a query is made to the system, as opposed to in eager learning, where the system tries to generalize the training data before receiving queries.

The main advantage gained in employing a lazy learning method, such as Case based reasoning [19], is that the target function will be approximated locally, such as in the $\mathrm{k}$-nearest neighbor algorithm. Because the target function is approximated locally for each query to the system, lazy learning systems can simultaneously solve multiple problems and deal successfully with changes in the problem domain. The disadvantages with lazy learning include the large space requirement to store the entire training dataset. Particularly noisy training data increases the case base unnecessarily, because no abstraction is made during the training phase. Another disadvantage is that lazy learning methods are usually slower to evaluate, though this is coupled with a faster training phase. In machine learning, instance-based learning or memory-based learning is a family of learning algorithms that, instead of performing explicit generalization, compare new problem instances with instances seen in training, which have been stored in memory. Instance-based learning is a kind of lazy learning.

Instance-based: It is called instance-based because it constructs hypotheses directly from the training instances themselves [9]. This means that the hypothesis complexity can grow with the data [9]: in the worst case, a hypothesis is a list of $n$ training items and classification takes $O(n)$. One advantage that instance-based learning has over other methods of machine learning is its ability to adapt its model to previously unseen data. Where other methods generally require the entire set of training data to be re-examined when one instance is changed, instance-based learners may simply store a new instance or throw an old instance away [32]. A simple example of an instance-based learning algorithm is the k-nearest neighbor algorithm. Daelemans and Van den Bosch describe variations of this algorithm for use in natural language processing (NLP), claiming that memory-based learning is both more psychologically realistic than other machine-learning schemes and practically effective [33].

Probably approximately correct learning (PAC) is a framework for mathematical analysis of machine learning. It was proposed in 1984 by Leslie Valiant [34]. In this framework, the learner receives samples and must select a generalization function (called the hypothesis) from a certain class of possible functions. The goal is that, with high probability (the "probably" part), the selected function will have low generalization error (the "approximately correct" part). The learner must be able to learn the concept given any arbitrary approximation ratio, probability of success, or distribution of the samples [34].

Support vector machines (SVMs) are a set of related supervised learning methods that analyze data and recognize patterns, used for classification and regression analysis. The original SVM algorithm was invented by Vladimir Vapnik and the current standard incarnation (soft margin) was proposed by Corinna Cortes and Vladimir Vapnik [35]. The standard SVM takes a set of input data, and predicts, for each given input, which of two possible classes the input is a member of, which makes the SVM a non-probabilistic binary linear classifier. Since an SVM is a classifier, then given a set of training examples, each marked as belonging to one of two categories, an SVM training algorithm builds a model that predicts whether a new example falls into one category or the other. Intuitively, an SVM model is a representation of the examples as points in space, mapped so that the examples of the separate categories are divided by a clear gap that is as wide as possible. New examples are then mapped into that same space and predicted to belong to a category based on which side of the gap they fall on [35-37]. More formally, a support vector machine constructs a hyper plane or set of hyper planes in a high or infinite dimensional space, which can be used for classification, regression or other tasks. Intuitively, a good separation is achieved by the hyper plane that has the largest distance to the nearest training data points of any class (so-called functional margin), since in general the larger the margin the lower the generalization error of the classifier [38]. Whereas the original problem may be stated in a finite dimensional space, it often happens that in that space the sets to be discriminated are not linearly separable. For this reason it was proposed that the original finite dimensional space be mapped into a much higher dimensional space presumably making the separation easier in that space [37] and [38].

\section{CONCLUSION}

This paper describes the best-known supervised techniques used in robot manipulators in relative detail. We should remark that our list of references is not a comprehensive list of papers discussing supervised methods. Our aim was to produce a critical review of the key ideas, rather than a simple list of all publications which had discussed or made use of those ideas. Despite this, we hope that the references cited cover the major theoretical issues, and provide access to the main branches of the literature dealing with such methods, guiding the researcher in interesting research directions. The paper shows that the key question when dealing with machine learning classification is not whether a learning algorithm is superior to others, but under which conditions a particular method can significantly outperform others on a given application problem.

\section{REFERENCES}

[1] T. M. Mitchell, "Machine Learning," McGraw Hill, 1997. 
[2] Ch. M. Bishop, "Pattern Recognition and Machine Learning," Springer, 2006.

[3] E. Alpaydın, "Introduction to Machine Learning (Adaptive Computation and Machine Learning)," MIT Press, 2004.

[4] S. Abney, "Semi supervised Learning for Computational Linguistics", Chapman \& Hall/CRC, 2008

[5] B. Settles, "Active Learning Literature Survey", Computer Sciences Technical Report 1648," University of Wisconsin-Madison, 2009, retrieved 2010-09-14.

[6] B. Taskar, V. Chatalbashev, D. Koller, C. Guestrin, "Learning Structured Prediction Models: A Large Margin Approach," ICML '05 Proceedings of the 22nd international conference on Machine learning, ISBN: 1-59593-180-5.

[7] Tie-Yan Liu, "Learning to Rank for Information Retrieval, Foundations and Trends in Information Retrieval," vol. 3, no. 3, 2009, pp. 225-331, ISBN 978-1-60198-244-5.

[8] G. I. Webb, J. Boughton and Z. Wang, "Not So Naive Bayes: Aggregating One-Dependence Estimators," Machine Learning, vol. 58, no. 1, 2005. Netherlands: Springer, pp. 5-24.

[9] S. Russell and P. Norvig, "Artificial Intelligence A Modern Approach," Prints hall, 2003.

[10] E. Bryson, Yu-Chi Ho, "Applied optimal control: optimization, estimation, and control," 1969, Blaisdell Publishing Company or Xerox College Publishing. pp. 481.

[11] L. A. Foreman, A. F. M. Smith and I. W. Evett, “ Bayesian analysis of deoxyribonucleic acid profiling data in forensic identification applications (with discussion)," Journal of the Royal Statistical Society, Series A, vol. 160, 1997, pp. 429-469.

[12] J. M. Bernardo, "A Bayesian mathematical statistics prior," ICOTS, vol. 7, 2006.

[13] H. Zhang, "The Optimality of Naive Bayes," FLAIRS, 2004

[14] R. Caruana and A. Niculescu-Mizil, "An empirical comparison of supervised learning algorithms," Proceedings of the 23rd international conference on Machine learning, 2006.

[15] G. H. John and P. Langley, "Estimating Continuous Distributions in Bayesian Classifiers", Proceedings of the Eleventh Conference on Uncertainty in Artificial Intelligence, 1995, pp. 338-345. Morgan Kaufmann, San Mateo.

[16] I. Ben-Gal, "Bayesian Networks", In Ruggeri, Fabrizio; Kennett, Ron S.; Faltin, Frederick W. Encyclopedia of Statistics in Quality and Reliability," John Wiley \&Sons, ISBN 978-0-470-01861-3, 2007.

[17] M. E. Borsuk, "Ecological informatics: Bayesian networks In Jørgensen, Sven Erik, Fath, Brian. Encyclopedia of Ecology,” Elsevier, ISBN 978-0-444-52033-3, 2008.

[18] J. Pearl, "Probabilistic Reasoning in Intelligent Systems," Networks of Plausible Inference. Representation and Reasoning Series (2nd printing ed.), 1988, San Francisco, California: Morgan

[19] A. Aamodt and E. Plaza, "Case-Based Reasoning: Foundational Issues, Methodological Variations, and System Approaches," Artificial Intelligence Communications, vol. 7 no. 1, 1994, pp. 39-52.

[20] T. Hastie, R. Tibshirani, J. H. Friedman, " The elements of statistical learning," Data mining, inference, and prediction, 2001, New York: Springer Verlag.

[21] L. Rokach, O. Maimon, "Top-down induction of decision trees classifiers-a survey," IEEE Transactions on Systems, Man, and Cybernetics, Part C vol. 35, 2005, pp. 476-487.

[22] S. H. Muggleton, "Inductive Logic Programming,"New Generation Computing, vol. 8, no. 4, 1991, pp. 295-318.

[23] S. H. Muggleton and L. De Raedt, "Inductive logic programming: Theory and methods," Journal of Logic Programming, vol. 19, 20, 1994, pp. 629-679.

[24] H. Bayraktar and F. S. Turalioglu, "A Kriging-based approach for locating a sampling site-in the assessment of air quality," SERRA vol.19, no.4, DOI 10.1007/s00477-005-0234-8, 2005, pp. 301-305.

[25] X. Emery, "Simple and Ordinary Kriging Multigaussian Kriging for Estimating recoverable Reserves," Mathematical Geology, vol. 37, no. 3, 2005, pp. 295-319.
[26] H. R. Madala and A. G. Ivakhnenko, "Inductive Learning Algorithms for Complex Systems Modeling," CRC Press, Boca Raton, 1994.

[27] S. J. Farlow, "Self-Organizing Methods in Modeling: GMDH Type Algorithms," New-York, Bazel: Marcel Decker Inc., vol. 350, 1984,

[28] A. G. Ivakhnenko, "Polynomial Theory of Complex System," IEEE Trans. on Systems, Man and Cybernetics, vol. SMC-1, no. 4, Oct. 1971, pp. 364-378.

[29] K. Narendra, M. A. L. Thathachar, "learning automata - a survey," IEEE Transactions on Systems, Man, and Cybernetics, vol. SMC-4, no. 4, July 1974 , pp. 323-334.

[30] M. L'vovich and T. Setlin, "Automaton Theory and the Modeling of Biological Systems," New York and London, Academic Press, 1973.

[31] L. P. Kaelbling, M. L. Littman; A. W. Moore, "Reinforcement Learning: A Survey," Journal of Artificial Intelligence Research vol. 4 1996, pp. 237-285.

[32] W. Daelemans and A. van den Bosch, "Memory-Based Language," Processing. Cambridge University Press, 2005.

[33] D. Bremner, E. Demaine, J. Erickson, J. Iacono, S. Langerman, P. Morin, G. Toussaint, "Output-sensitive algorithms for computing nearest-neighbor decision boundaries". Discrete and Computational Geometry vol. 33, no. 4, 2005, pp. 593-604.

[34] L. Valiant," A theory of the learnable Communications of the ACM, vol. $27,1984$.

[35] C. Cortes and V. Vapnik, "Support-Vector Networks," Machine Learning, vol. 20, 1995

[36] D. Meyer, F. Leisch, and K. Hornik, "The support vector machine under test," Neurocomputing vol. 55, no. 1-2, 2003, pp. 169-186.

[37] C. Cortes and V. Vapnik, "Support-Vector Networks," Machine Learning, vol. 20, 1995.

[38] M. Aizerman, E. Braverman, and L. Rozonoer, "Theoretical foundations of the potential function method in pattern recognition learning," Automation and Remote Control vol. 25, 1964, pp. 821-837.

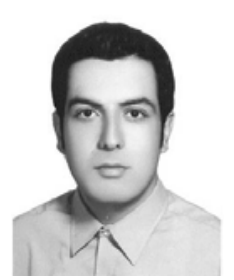

Hadi Hormozi is currently serving as a senior Lecturer at Faculty of ICT, Islamic Azad University, Buinzahra branch. He obtained his

B.Sc. from Azad University of Shahriar, Shahriar in 2006 and M.Sc. from Azad University of Arak, Arak in 2010 both in Software Engineering. His current interests include Machine Learning, Grid Computing and Cloud Computing.

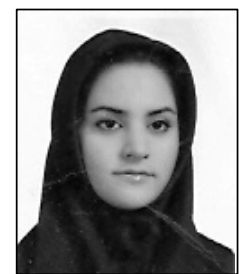

Elham Hormozi received M.Sc. in Information Technology from Mazandaran University of Science and Technology (MUST) in 2011. She received B.Sc. in Software Engineering from Azad University of Yazd, Yazd, 2007. His research interests are in the areas Cloud Computing, Machine Leaning and their applications.

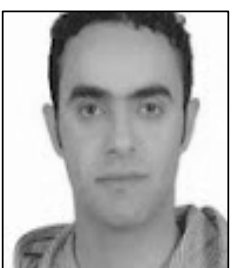

Hamed Rahimi Nohooji is currently PhD Student at University of Pisa, Italy. He received his B.Sc. in Mechanical Engineering from Azad University of Semnan, Semnan, 2006 and M.Sc. in Mechanical Engineering from Iran University of Science and Technology (IUST), Tehran, 2009. His research interests are Dynamics and Control, Robotics, Bio-Engineering, Bio Mechanics and Tribology. 